\title{
Reduction of the Retransmission Delay for Heterogeneous Devices in Dynamic Opportunistic Device-to-device Network
}

\author{
Sixuan Chen ${ }^{1}$, Weixia Zou ${ }^{1,2^{*}}$, Xuefeng Liu ${ }^{1}$, Yang Zhao ${ }^{1}$, and Zheng Zhou ${ }^{1}$ \\ ${ }^{1}$ Key Laboratory of Universal Wireless Communications, Ministry of Education, University of Information and \\ Communication Engineering, Beijing University of Posts and Telecommunications, \\ Beijing 100876 - CHINA \\ [e-mail: \{chensixuan2131, liuxfeng6, buptzy93\}@163.com; zzhou@bupt.edu.cn] \\ ${ }^{2}$ State Key Laboratory of Millimeter Waves, School of Engineering, Southeast University \\ Nanjing 210096 - CHINA \\ [e-mail: zwx0218@bupt.edu.cn] \\ *Corresponding author: Weixia Zou
}

Received October 10, 2017; revised March 5, 2018; revised April 16, 2018; accepted May 5, 2018; published October 31, 2018

\begin{abstract}
The dynamic opportunistic device-to-device (DO-D2D) network will frequently emerge in the fifth generation (5G) wireless communication due to high-density and fast-moving mobile devices. In order to improve the Quality of Experience (QoE) of users with different computing capacity devices in the DO-D2D network, in this paper, we focus on the study of how to reduce the packets retransmission delay and satisfy heterogeneous devices. To select as many devices as possible to transmit simultaneously without interference, the concurrent transmitters-selecting algorithm is firstly put forward. It jointly considers the number of packets successfully received by each device and the device's connectivity. Then, to satisfy different devices' demands while primarily ensuring the base-layer packets successfully received by all the devices, the layer-cooperation instantly decodable network coding is presented, which is used to select transmission packets combination for each transmitter. Simulation results illustrate that there is an appreciable retransmission delay gain especially in the poor channel quality network compared to the traditional base-station (BS) retransmission algorithm. In addition, our proposed algorithms perform well to satisfy the different demands of users with heterogeneous devices.
\end{abstract}

Keywords: Dynamic opportunistic D2D network, heterogeneous devices, instantly decodable network coding, maximal clique problem, retransmission

This work was supported by NSFC (No. 61571055), fund of SKL of MMW (No. K201815), Important National Science \& Technology Specific Projects(2017ZX03001028). 


\section{Introduction}

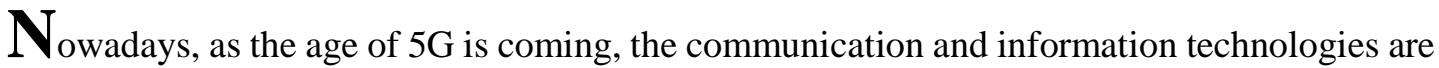
advancing sharply and the variety of smart devices is expanding universally, the traffic on the mobile network achieves explosive growth [1]. Video continues to be the major application generator for mobile data traffic growth, and it will account for 75 percent of global mobile data traffic by 2020 [2][3]. How to satisfy the users' Quality of Experience (QoE) demand has attracted more and more attention. QoE is a subjective measure of a customer's experiences with a service focuses on the entire service experience [4]. Network performance is one of the factors that effects QoE levels, which depends on time delays and packet losses [5]. Many reasons may cause packets loss, such as: moving, being obstructed and so on. Retransmission packets can improve reliability of transmission. Reducing the retransmission times with reliable transmission, by this way, the users' QoE may increase [6][7]. Device-to-device (D2D) communication is one of the key technologies in 5G which enables two nearby users to communicate directly. It can increase spectral efficiency and energy efficiency, help offload traffic from cellular networks, and reduce transmission delay [8]. So, D2D communication is a good choice to transmit video data stream.

The dynamic opportunistic device-to-device (DO-D2D) network is described by the characteristic of sparse network deployment, probabilistic node mobility and interruptible wireless links [9], which will cause instantaneously poor channel quality and increase the retransmission probability of packets. It is proved that network coding (NC) can be efficient for providing improved performance with respect to the reduction of retransmission delay [10][11]. Besides, the instantly decodable network coding (IDNC) has attracted a considerable amount of research in rencent years, which can provide instantaneously decoding of packets in receivers. Meanwhile, it is proved IDNC shows a propriety of great interest for real-time applications, which also can efficiently reduce the packets retransmission times in erasure network [12][13].

The reference [14] studied the dynamic D2D network with heterogeneous radio access. A new heterogeneous radio access network (RAN) architecture is proposed and by borrowing resources from D2D communication based mobile devices in a cloudlet to provide faster computing and lower latency for end-user devices. The works in [15] showed that how to find the optimal IDNC schedule for minimizing the overall completion time in point to multi-point (PMP) network, and proved it is computationally intractable due to the curse of dimensionality of dynamic programming. The authors in [16] proposed a local graph formulation that models the encoding step in partially connected systems. From the references, we know that in the D2D system it is also a NP-hard problem to find the minimum delay, and it is an intractable problem to find the transmission devices to minimize the total completion time.

However, erasure network and heterogeneous devices make it even more difficult to satisfy the users' QoE demands, because each device has different specifications and requires different stream data [17]. In addition, the existing indifference data processing retransmission scheme treats all terminals equally, which will cause the low-computing terminals have to face high waiting delay. How to satisfy the QoE demands of the medium and heterogeneous computing capacity terminals while minimizing the waiting delay of low-computing terminals is still a problem worth to study. In order to tackle this issue, we can exploit the advantages of scalable video coding (SVC) which can perfectly satisfy these different necessities [18]. SVC divides the data into different hierarchical order layers, and the decoding of high-level layer 
depending on the lower layers. Users can request a different number of layers based on their own requirements [19][20].

In this paper, we study how to reduce the retransmission times of packets while satisfying different demands of heterogeneous devices in DO-D2D network. The transmitter (e.g., a base-station (BS) or a device) wants to broadcast a set of $N$ source packets, which employs SVC to divide the data into several layers of different clarity. In an initial phase, the BS broadcasts the packets one after the other. Due to the erasure nature of the wireless medium, the diversity of received/lost packets is presented between receivers. Then, in the recovery phase, the transmitter (one or more devices) sends XOR-encoded packets to target multiple receivers. This phase is repeated until all devices successfully receive all wanted packets. In this recovery phase, we utilize D2D communication to retransmit packets. Devices can exchange their received packets with the neighbor devices which locate in their transmission range. Firstly, a heuristic transmission devices selection algorithm is proposed to select some concurrent transmitting devices as transmitters in one slot. Next, in order to select transmission packets combination for each transmitter, a layer cooperation IDNC algorithm is presented. These selected packets are coded with NC by each transmitter and then broadcasted to the devices which are in the transmission range of transmitters.

The rest of this paper is organized as follows: Section 2 depicts the system model and states the problem we want to solve. The heuristic transmission devices selecting algorithm and the improved algorithm of the case that all the devices want the same number of packets are presented in Section 3. In Section 4 we propose the layer cooperation IDNC algorithm to tackle different users' demands of heterogeneous devices and give a detailed introduction of constructing window to ensure the priority of base-layer successfully received by all users. Analysis of simulation and experimental results are described in Section 5. Finally, section 6 concludes the works we have done in this paper.

\section{System Analysis}

In this study, we consider a DO-D2D network consisting of $M\left(\mathcal{M}=\left\{D_{1}, \ldots, D_{M}\right\}\right)$ devices. The BS has $N\left(\mathcal{N}=\left\{P_{1}, \ldots, P_{N}\right\}\right)$ source packets need to be broadcasted. Due to the heterogeneous computing capacity of devices, they request different qualities of videos. However, a wanted packet by one device may unwanted by its neighbors. By using SVC, the $N$ source packets are divided into $l\left(\mathcal{L}_{1}, \mathcal{L}_{2}, \ldots, \mathcal{L}_{l}\right)$ layers, and each layer contains one or more packets. With the increasing number of received decoding video layers at each device, the video quality is gradually improving. And the number of video layers that each device requires depends on its decoding capability.

The meeting-probability between nodes is uncertain since the randomized moving directions and different moving speeds. However, any two devices can communicate with each other by one-hop or multi-hop. In the period $(t, t+\Delta t)$, we assume that the network topology is unchanged. So the connected matrix of devices is assumed to be invariant in period $(t, t+\Delta t)$ and updated at intervals $\Delta t$.

In the initial BS broadcast phase, all the devices listen the transmitted packets. Since the erasure nature of channel, three sets may be fed back to BS:

The Has set $\mathcal{H}_{i}$ is defined as the set of packets successfully received by device $i$ (denoted by $\left.D_{i}\right)$;

The Lost and Not-Want set $\mathcal{L} \mathcal{N} \mathcal{W}_{i}$ is the set of packets which is not successfully received by $D_{i}$, but don't want to receive; 
The Lost and Want set $\mathcal{L} \mathcal{W}_{i}$ is the set of packets that $D_{i}$ has not yet received, but want to receive.

After each transmission, BS will receive feedbacks from devices and update the $M \times N$ feedback matrix $\mathbf{F}=\left[f_{i, j}\right]$. The feedback matrix is shown in formula (1):

$$
f_{i, j}=\left\{\begin{array}{l}
0, \text { if } P_{j} \in \mathcal{H} \Gamma_{i} \\
1, \text { if } P_{j} \in \mathcal{L} \mathcal{W}_{i} \\
-1, \text { if } P_{j} \in \mathcal{L} \mathcal{N} W_{i}
\end{array}\right.
$$

Where $P_{j}$ denotes the packet $j$. After the first BS broadcast phase, we assume an arbitrary packet can be found at least in one device. And next, by using the matrix $\mathbf{F}$, the transmitters can be selected from the $M$ devices. In each transmission, the transmitters encode some of the packets together using linear coefficients from $F_{q}$ Galois field. When the total number of ' 1 ' ' in row $i$ of matrix $\mathbf{F}$ is zero that means $D_{i}$ has successfully received all the packets it needs. The details will be stated in section 3. The system model is illustrated in Fig. 1, in the first phase, BS broadcasts all the packets to devices. Since the channel's erasure nature, some devices haven't successfully received their wanted packets during the first phase, and they will request the lost packets from other devices. In the second retransmission phase, one or more devices are selected as the transmitter to process a D2D communication for exchange packets. For example, in Fig. 1, we select $D_{2}$ and $D_{6}$ as the transmitters.

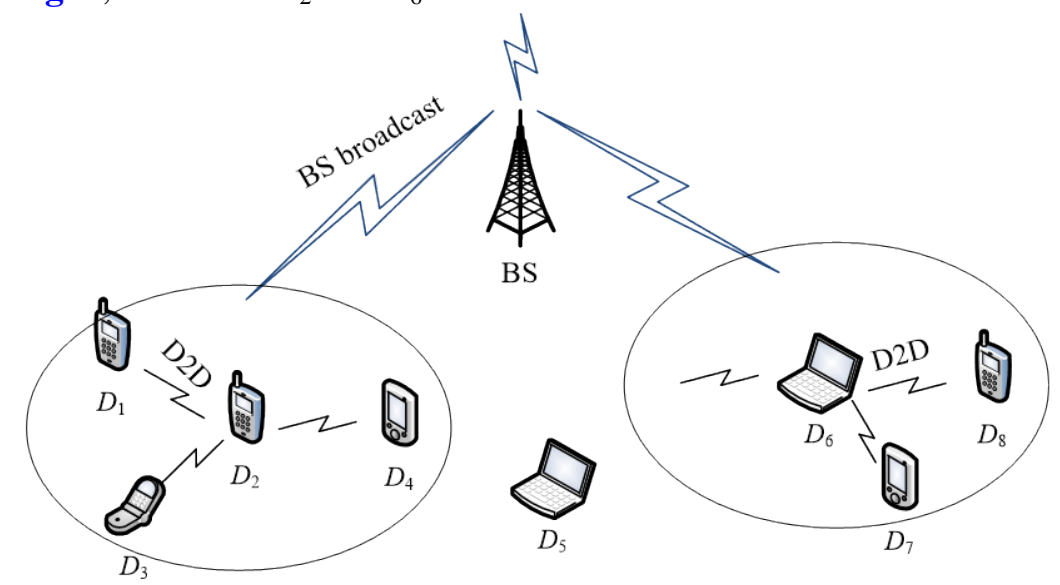

Fig. 1. The system model

In addition, we assume that we have obtained the demand table of all the devices. For example, the demand table is shown in Table 1.

Table 1. The required layers for each device

\begin{tabular}{|c|c|c|c|c|c|c|c|}
\hline & \multicolumn{3}{|c|}{$\mathcal{L}_{1}$} & \multicolumn{2}{|c|}{$\mathcal{L}_{2}$} & \multicolumn{2}{|c|}{$\mathcal{L}_{3}$} \\
\hline & $P_{1}$ & $P_{2}$ & $P_{3}$ & $P_{4}$ & $P_{5}$ & $P_{6}$ & $P_{7}$ \\
\hline$D_{1}$ & 1 & 1 & 1 & 1 & 1 & -1 & -1 \\
\hline$D_{2}$ & 1 & 1 & 1 & -1 & -1 & -1 & -1 \\
\hline$D_{3}$ & 1 & 1 & 1 & 1 & 1 & 1 & 1 \\
\hline$D_{4}$ & 1 & 1 & 1 & 1 & 1 & -1 & -1 \\
\hline
\end{tabular}


There are three layers: $\mathcal{L}_{1}$ is the base layer, which has 3 packets; $\mathcal{L}_{2}$ and $\mathcal{L}_{3}$ are enhancement layers. In this table, ' 1 ' represents the device want to get this packet, and ' -1 ' represents does not want to get, while ' $\mathcal{W} \mathcal{D}_{i}$ ' represents the set of packets wanted by device $i$. And $\mathcal{D}_{W}=\left\{D_{i} \mid\right.$ $\mathcal{W D}_{i} \neq \Phi, i \in M$ \} denotes a set of devices which have not successfully received all the wanted packets. At each transmission, some of the devices are selected as transmitters. Then, at each transmitter some of the packets will be chosen to perform network coding and will be broadcasted. Our purpose is to minimize the total times that all the devices successfully receive their wanted packets. Can we find the perfect transmission scheme?We first introduce some related concepts of the hypergraph theory. A hypergraph $\mathcal{H}_{G}$ is defined by a pair $\left(\mathcal{V}_{\mathscr{H}}\right.$, $\mathcal{E}_{\mathcal{H}}$ ), where $\mathcal{V}_{\mathcal{H}}$ is the set of vertices, and $\mathcal{E}_{\mathcal{H}}$ is the set of hyperedges [21]. A hypergraph is non-uniform if it has at least two edge types. A size- $k_{\mathcal{H}}$ strong coloring of $\mathcal{H}_{G}$ is a partition of $\mathcal{V}_{\mathcal{H}}$ into $k_{\mathcal{H}}$ subsets $\left(S_{1}, S_{2}, \ldots, S_{k_{\mathcal{H}}}\right)$. In other words, every color appears at most once in every hyperedge, i.e. $\left|S_{i} \cap e_{j}\right| \leq 1, \forall e_{j} \subset \mathcal{E}_{\mathcal{H}}$ [22].

For our question, we let $\{x\}_{i=1}^{k_{\mathcal{H}}}$ be the set of packets corresponding to $\{s\}_{i=1}^{k_{\mathcal{H}}}$, each $x_{i}$ is a NC scheme. $\mathcal{L} \mathcal{W}_{m}$ is the want packets set of receiver $m$, which corresponds to $e_{j}$. So if $\mid S_{i} \cap$ $\mathcal{L} \mathcal{W}_{m} \mid=1, \forall i \in\left\{1, \ldots, k_{\mathcal{H}}\right\}, m \in\{1, \ldots, M\}$, we can enable every receivers to decode a want packet at each transmission. This is a non-uniform hypergraph strong coloring problem.

Lemma 1 ([23]): A simple hypergraph $\mathcal{H}_{G}$ (i.e. every two distinct edges have at most one common vertex), and with minimum edge-cardinality $n$, and $\sum_{e \in \mathcal{E}} r^{1-|e|} \leq c \sqrt{n}$, for some absolute constant $c>0$, then $\mathcal{H}_{G}$ is $r$-colorable.

Lemma 2 ([24]): Every strong $k_{\mathscr{H}}$-colorable hypergraph is a $k_{\mathcal{H}}$-colorable hypergraph, but not vice-versa.

In conclusion, we showed that the problem of non-uniform hypergraph strong coloring problem is NP-hard. So it is hard to find the transmitting packets combinations select strategy to minimize the total number of retransmissions in the dynamic heterogeneous D2D network with erasure channels.

\section{Transmission Devices Selecting Strategy Design}

During the second transmission phase, we use the D2D directly communication to retransmit the lost packets. The coverage range between the transmitting devices may overlap, and the devices in the overlapping range can monitor two or more transmitters at the same time called the interfered devices. Like Fig. 2 shows, $\left\{D_{1}, D_{2}\right\}$ are selected as transmitters. $\left\{D_{3}, D_{4}, D_{5}\right\}$ can receive packets from $D_{1} . D_{5}$ is an interfered device located in the overlapping region of $\left\{D_{1}, D_{2}\right\}$, and $\left\{D_{8}, D_{9}, D_{10}, D_{11}\right\}$ are out of the transmitting range of $\left\{D_{1}, D_{2}\right\}$.

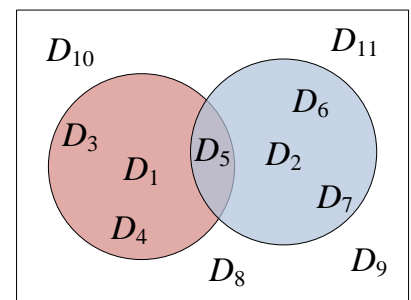

Fig. 2. Classification of devices 
Both the interfered devices and devices which out of transmitting range cannot decode a packet instantly in this transmission. Hence, the problem turns into how to maximize the number of concurrent transmission devices and meanwhile minimize the number of interfered devices.

In this section, we consider the case that all the devices want the same number of packets and we propose the heuristic non-interference transmission devices selecting algorithm.Firstly, we propose a transmitters-selecting algorithm depending on maximal weight of transmission devices (DMWT), and the details are stated in section 3.1.

\subsection{Transmitters-Selecting Algorithm}

Aim to reduce the total time of all the devices successfully receiving the wanted packets, some devices are selected as the transmitters, which can concurrently transmit packets to the devices located in their transmission regions. We use $\mathcal{K}$ to store the selected transmission devices. $\mathbf{C}$ denotes the adjacent matrix of devices and will be updated at interval $\Delta t$. How to select the transmission devices, we firstly set a weight for each device, as formula (2) shows:

$$
w_{i}=\left|\mathcal{H}_{i}\right| \times \sum_{j=1}^{d_{i}}\left(1-p_{i j}\right)
$$

Where $\left|\mathcal{H}_{i}\right|$ is the number of packets successfully received by device $i, d_{i}$ is the degree of connectivity of device $i$, and $p_{i j}$ is the erasure probability of device $i$ to device $j$.

At first, search the maximal weight device as the transmitter and put it in $\mathcal{K}$. Then, find the devices connected to the devices in $\mathcal{K}$ from matrix $\mathbf{C}$, i.e. the devices located in the transmission region of the transmitter in $\mathbf{C}$, and put it in $\mathcal{K}^{\prime}$ as the first level connected devices. By the same way, find the second level connected devices which are connected to the devices in $\mathcal{K}^{\prime}$, and put it in $\mathcal{K}^{\prime \prime}$. The devices in $\mathcal{K}^{\prime \prime}$ cannot be selected as transmitters, otherwise they will interfere the transmissions. The details will be stated in Example 1. Let $\mathcal{D}$ stores a set of $M$ devices, and $\mathcal{Q}=\mathcal{D}-\mathcal{K}-\mathcal{K}^{\prime}-\mathcal{K}^{\prime \prime}$ represents the candidate devices. These steps are repeated until there is no candidate device. The details of this algorithm have shown in Fig. 3.

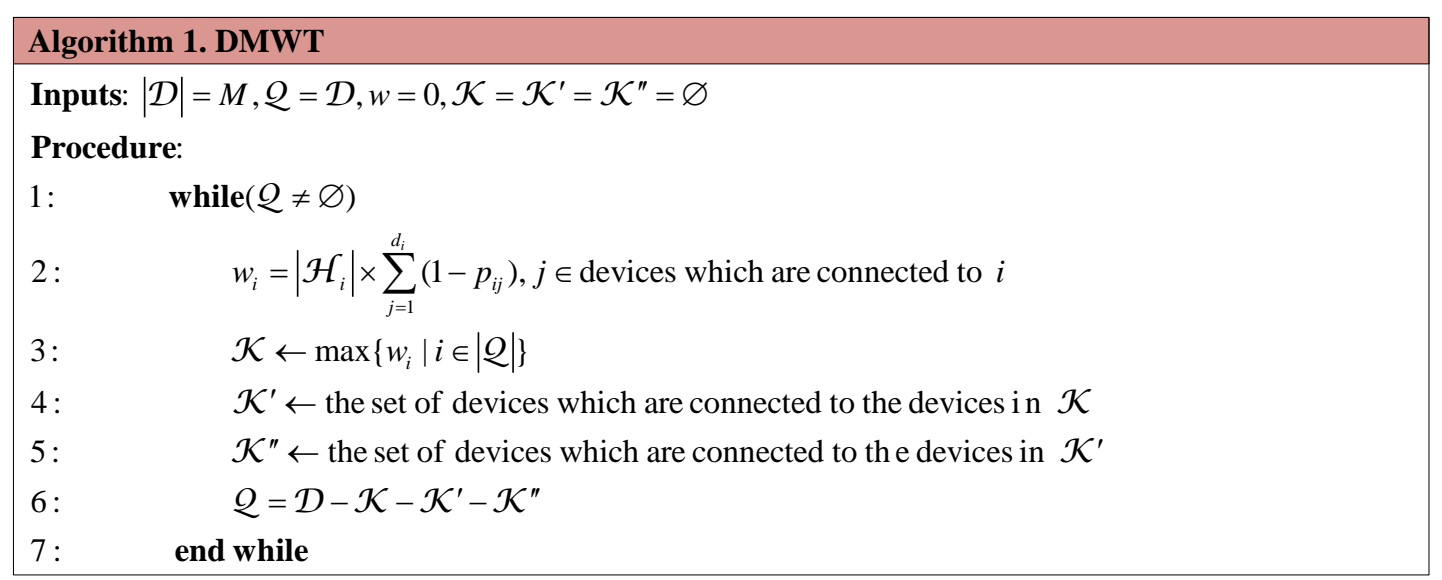

Fig. 3. The procedure of DMWT algorithm 


\section{Example 1:}

We consider a network with 9 devices and 10 packets, and assume all the devices want the 10 packets. We assume they have the same erasure probability between either one of the devices in this example. The adjacent matrix has shown in Table 2 and the feedback matrix has stated in Table 3.

Table 2. The adjacent matrix between devices

\begin{tabular}{|l|lllllllll|}
\hline & $D_{1}$ & $D_{2}$ & $D_{3}$ & $D_{4}$ & $D_{5}$ & $D_{6}$ & $D_{7}$ & $D_{8}$ & $D_{9}$ \\
\hline \hline$D_{1}$ & 0 & 0 & 1 & 1 & 1 & 0 & 1 & 0 & 0 \\
$D_{2}$ & 0 & 0 & 0 & 0 & 1 & 1 & 1 & 0 & 0 \\
$D_{3}$ & 1 & 0 & 0 & 0 & 0 & 0 & 0 & 1 & 0 \\
$D_{4}$ & 1 & 0 & 0 & 0 & 0 & 0 & 0 & 0 & 1 \\
$D_{5}$ & 1 & 1 & 0 & 0 & 0 & 0 & 1 & 0 & 0 \\
$D_{6}$ & 0 & 1 & 0 & 0 & 0 & 0 & 1 & 0 & 0 \\
$D_{7}$ & 1 & 1 & 0 & 0 & 1 & 1 & 0 & 0 & 0 \\
$D_{8}$ & 0 & 0 & 1 & 0 & 0 & 0 & 0 & 0 & 1 \\
$D_{9}$ & 0 & 0 & 0 & 1 & 0 & 0 & 0 & 1 & 0 \\
\hline
\end{tabular}

Table 3. The feedback matrix of devices

\begin{tabular}{|l|llllllllll|}
\hline & $P_{1}$ & $P_{2}$ & $P_{3}$ & $P_{4}$ & $P_{5}$ & $P_{6}$ & $P_{7}$ & $P_{8}$ & $P_{9}$ & $P_{10}$ \\
\hline \hline$D_{1}$ & 1 & 1 & 1 & 1 & 0 & 0 & 1 & 0 & 0 & 1 \\
$D_{2}$ & 0 & 1 & 1 & 1 & 1 & 1 & 1 & 0 & 0 & 1 \\
$D_{3}$ & 0 & 0 & 0 & 0 & 1 & 0 & 0 & 0 & 0 & 1 \\
$D_{4}$ & 1 & 1 & 1 & 0 & 1 & 1 & 0 & 0 & 1 & 1 \\
$D_{5}$ & 1 & 1 & 0 & 0 & 0 & 0 & 0 & 1 & 1 & 1 \\
$D_{6}$ & 1 & 1 & 1 & 0 & 0 & 0 & 0 & 1 & 0 & 1 \\
$D_{7}$ & 0 & 0 & 1 & 0 & 0 & 0 & 0 & 1 & 0 & 1 \\
$D_{8}$ & 0 & 1 & 0 & 0 & 0 & 0 & 1 & 0 & 0 & 0 \\
$D_{9}$ & 1 & 0 & 1 & 0 & 0 & 0 & 1 & 0 & 0 & 1 \\
\hline
\end{tabular}

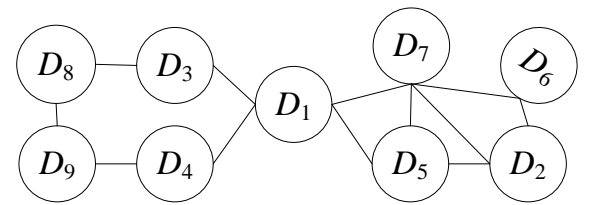

Fig. 4. The connected graph according to Table 2

Step 1: The device with the maximal weight is $D_{7}$ and put it in $\mathcal{K}$. And we can find the first level connected devices and the second level connected devices sequentially. So $\mathcal{K}=\left\{D_{7}\right\}$, $\mathcal{K}^{\prime}=\left\{D_{1}, D_{2}, D_{5}, D_{6}\right\}, \mathcal{K}^{\prime \prime}=\left\{D_{3}, D_{4}, D_{5}, D_{7}\right\} \cup\left\{D_{5}, D_{6}, D_{7}\right\} \cup\left\{D_{1}, D_{2}, D_{7}\right\} \cup\left\{D_{2}, D_{7}\right\}=\left\{D_{1}, D_{2}\right.$, $\left.D_{3}, D_{4}, D_{5}, D_{6}, D_{7}\right\}, \mathcal{Q}=\mathcal{D}-\mathcal{K}^{\prime}-\mathcal{K}^{\prime \prime}=\left\{D_{8}, D_{9}\right\}$. Here, $D_{7}$ is the transmitter, and $\left\{D_{1}, D_{2}, D_{5}, D_{6}\right\}$ are the receivers of $D_{7} . D_{3}, D_{4}$ will not be selected as transmitters to concurrently transmit packets with $D_{7}$, that is to avoid interferring with the transmission of $D_{7} \rightarrow D_{1}$;

Step 2: After step 1, we obtain a sub-graph, which is constructed by the devices in $\mathcal{Q}$ and the connect edge between them. Then, find the maximal weight device from the sub-graph, and add it to the set $\mathcal{K}$. Finally, update $\mathcal{K}^{\prime}, \mathcal{K}^{\prime \prime}, \mathcal{Q}$ as step 1 . Here, $\mathcal{K}=\left\{D_{7}, D_{8}\right\}, \mathcal{Q}=\{\Phi\}$; 
Step 3: If $\mathcal{Q} \neq\{\Phi\}$, return to the step 2. Repeat the previous steps until $\mathcal{Q}=\{\Phi\}$.

According to algorithm 1, the devices are divided into three categories: transmission devices, receivers and out of range devices. This algorithm has an obvious deficiency: after many times of retransmissions, most of devices have successfully received the wanted package, but a few devices are still requesting packets, i.e. $D_{W} \neq\{\Phi\}$, and their locations are maybe sparsely distributed. We use Rec denotes the sub-feedback matrix, of which the rows is the receivers corresponding to the selected transmission devices in $k$. In this situation, all the receivers covered by the transmission devices selected through algorithm 1 may have already successfully received. However, there are some un-successfully received devices located out of the transmitting range. Take Fig. 4 for example, we assume the only un-successfully received device is $D_{4}$, if select the transmitter according to the above algorithm, we can get the transmitter $D_{7}, D_{8}$. So in this transmission, $D_{4}$ cannot decode an useful packet. This transmission is considered as invalid retransmission and increases the number of retransmission times. Therefore, we propose an improved transmitters-selecting algorithm.

\subsection{Improved Transmitters-Selecting Algorithm}

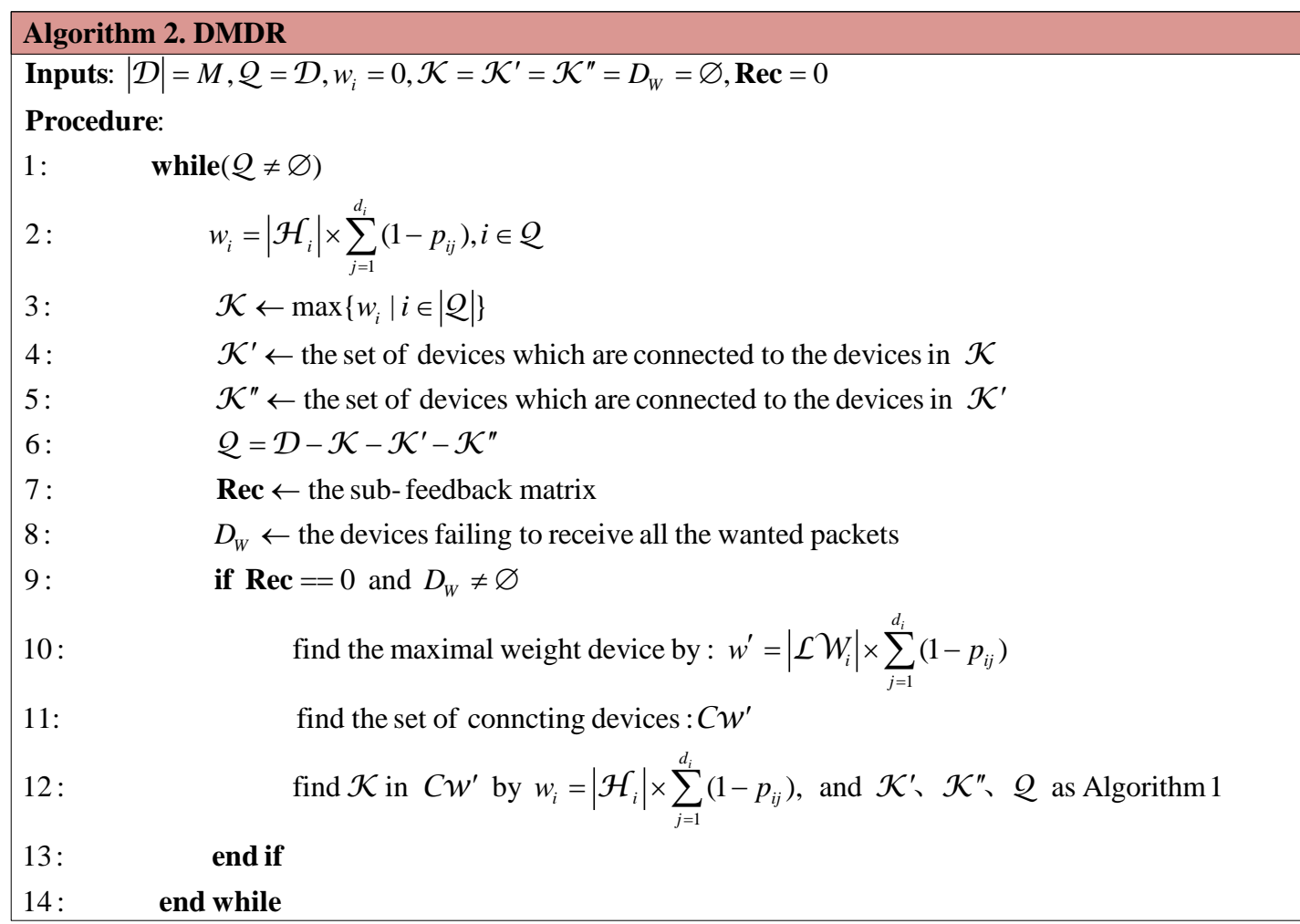

Fig. 5. The procedure of DMDR algorithm

When the above situation occurs, i.e. the number of elements in Rec which are equal to 1 is zero and $D_{W} \neq\{\Phi\}$, and an improved algorithm based on algorithm 1 is proposed to tackle this problem. The improved algorithm is jointly considering the maximal weitht of transmission devices and the maximal delay of receivers, which is called DMDR Algorithm. The weight is reset as formula (3), and then find the device with maximal weight. The device which has the maximal weitht may need more times to complete received all the wanted packets. The overall complete times of all devices depending on the device which need the most retransmission 
times. So, it's better to give this device priority to be selected as receiver in this transmission.

Firstly, find the device with maximal weight. Secondly, find the devices connected to the maximal weight device and store them in $C w^{\prime}$. Then, compute the weight of devices in $C w^{\prime}$ by using the formula (2).

$$
w^{\prime}=\left|\mathcal{L} \mathcal{W}_{i}\right| \times \sum_{j=1}^{d_{i}}\left(1-p_{i j}\right)
$$

Hence, we design an improved transmission devices select algorithm as Fig. 5 shows.

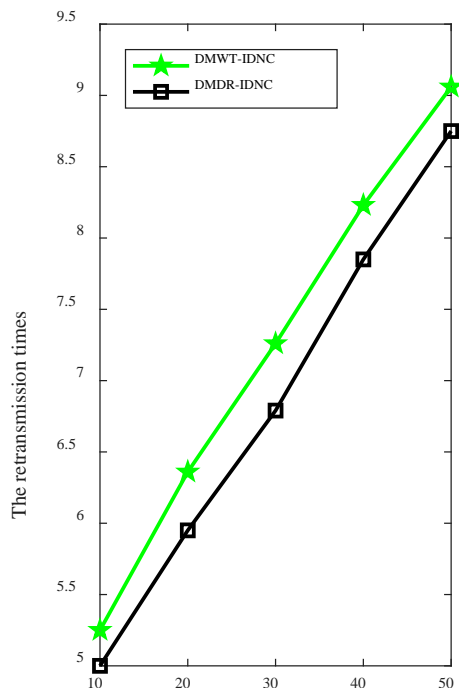

(a) The number of devices

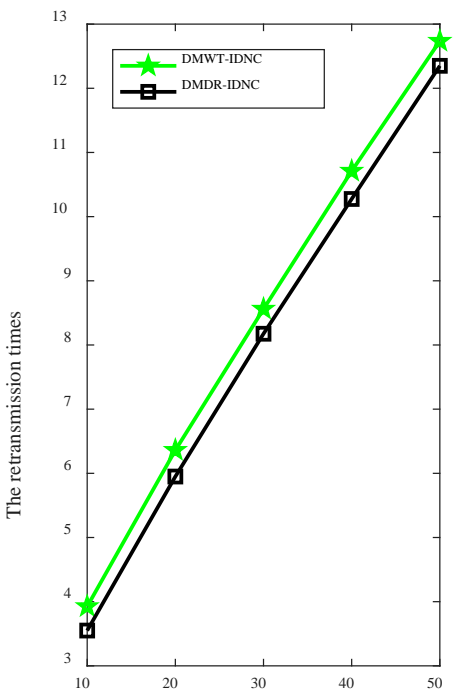

(b) The number of packets

Fig. 6. (a) Retransmission times versus the number of devices for a network of 20 packets; (b) Retransmission times versus the number of packets with the network of 20 devices with average moving speed: $8 \mathrm{~m} / \mathrm{s}$, BS to device channel erasure probability: 0.15 , connectivity:0.8

Then, we compare the DMWT algorithm with DMDR algorithm, and the simulation results are illustrated in Fig. 6 and Fig. 7. The packets-selecting algorithm uses the IDNC algorithm in this simulation, which is briefly introduced in section 4. Combine this two transmitters-selecting algorithms with IDNC, and denote them as DMWT-IDNC and DMDR-IDNC respectively. In Fig. 6, the connectivity is set to 0.8, with the number of devices increase and packets increase, the two algorithms present approximately performance, the DMDR-IDNC algorithm performs a little better than the DMWT-IDNC algorithm. However, the performances of the two algorithms are affected by the factor of connectivity to some extent as Fig. 7 shows. Fig. 7 illustrates that when there is a well conncetivity in the network $(>=0.6)$, DMWT-IDNC algorithm can perform much better than it in network with low connectivity, which can reach a similar retransmission times with DMDR-IDNC algorithm. In order to avoid interference between transmitters, with the increasing of connectivity, the selected number of concurrent transmitting devices will decrease in one transmission, so the total retransmission times will perform a little rise again after a fall. However, we can obtain that the DMDR-IDNC algorithm performs a obviously better retransmission delay gain than the DMWT-IDNC algorithm when the network with low or moderate connectivity (0.15 to 0.5), so in the following, we take the DMDR-IDNC to carry out a further analysis and denote it 
as DI algorithm.

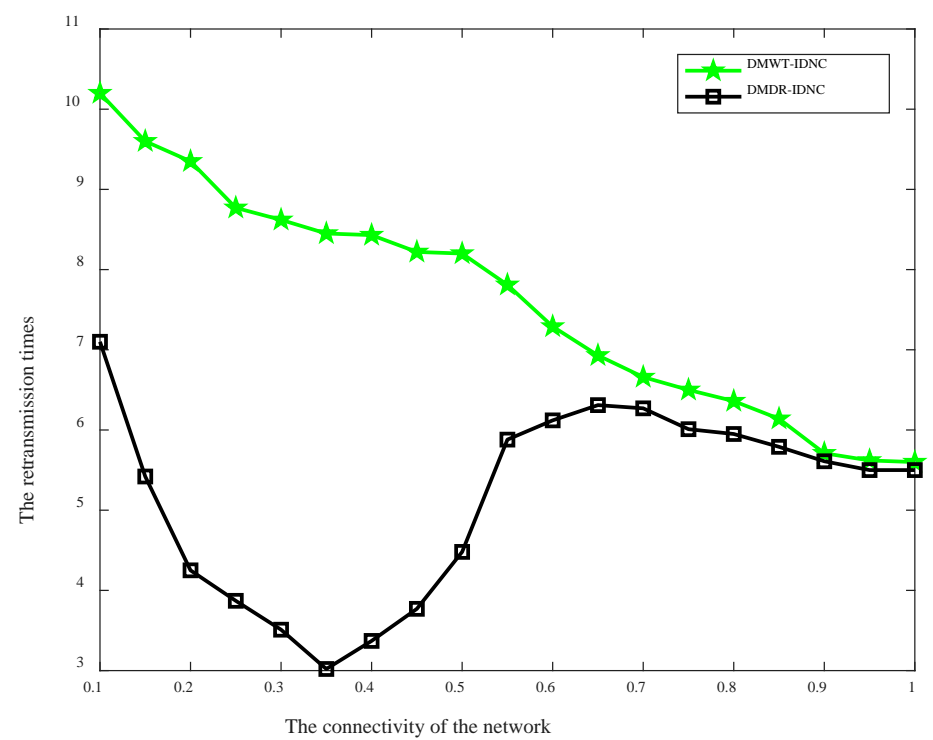

Fig. 7. Comparring the Retransmission times of DMWT-IDNC algorithm with DMDR-IDNC algorithm versus connectivity with a network of 20 packets, 20 devices, average moving speed: $8 \mathrm{~m} / \mathrm{s}$, BS to device channel erasure probability: 0.15

\section{Transmission Packets Selecting Algorithm Design}

\subsection{Layer Cooperation IDNC Algorithm}

In reference [25], the writers proposed the IDNC algorithm in PMP network. This algorithm provides a novel network coding scheme to instantly decode for receivers. The IDNC graph $\mathcal{G}_{\rho}\left(\mathcal{V}_{\rho}, \mathcal{E}_{\rho}\right)$ can be illustrated from the feedback matrix. $\mathcal{V}_{\rho}$ is the set of vertexes corresponding to the elements not equal to'0'in feedback matrix. The two vertexes $v_{i j}, v_{k l}$ are connected if they satisfy one of the two conditions, $C 1: j=l ; C 2: \mathrm{j} \in \mathcal{H}_{k}$ and $l \in \mathcal{H}_{i}$.

Then search the maximal clique [26][27] from the IDNC graph to obtain the transmission packets combination. Much more details are available in reference [25].

In this section, we consider the case that the heterogeneous computing capabilities of devices require different number of layers. In order to find the transmission packets, we propose a layer cooperation IDNC (LCID) algorithm. First, we construct the layer cooperation IDNC Graph $\left(\mathrm{G}_{L C-I D N C}(\mathcal{V}, \mathcal{E})\right)$. Here, $\mathcal{V}$ is a set of vertices corresponding to the element in feedback matrix which is equal to ' 1 '. We assume the device as a receiver will not store the unwanted received packets. We define $v_{l j p}^{i} \in V$, of which $i$ represents the transmitter $i, j$ represents one of the receivers in the transmission range of transmitter $i, l$ represents the wanted-layers of device $j$. Two vertices $v_{l j p}^{i}$ and $v_{l l^{\prime} p^{\prime}{ }^{\prime}}^{i^{\prime}}$ are connected by an edge in graph $\mathrm{G}_{L C-I D N C}$ $(\mathcal{V}, \mathcal{E})$ if they satisfy the following three conditions:

1) $i=i^{\prime}$, this represents that the two different devices can directly communicate to the same device;

2) $\quad l=l^{\prime}$, this represents that the devices want the same layer packets;

3) $P=P^{\prime}$ or $\left(P \in \mathcal{H}_{j^{\prime}} \& P^{\prime} \in \mathcal{H}_{j}\right)$. $P=P^{\prime}$ represents different vertices lose the same packets; 
$P \in \mathcal{H}_{j^{\prime}} \& P^{\prime} \in \mathcal{H}_{j}$ represent the packet one device wants is owed by the other devices.

\section{Example 2:}

We consider four devices and assume they were connected to each other, seven packets which are separated to 3 layers as Table $\mathbf{1}$. And the layers required for each device also has shown in Table 1, and the feedback matrix is presented as Table 4.

Table 4. Feedback matrix

\begin{tabular}{|c|c|c|c|c|c|c|c|}
\hline & $P_{1}$ & $P_{2}$ & $P_{3}$ & $P_{4}$ & $P_{5}$ & $P_{6}$ & $P_{7}$ \\
\hline \hline$D_{1}$ & 1 & 0 & 1 & 0 & 1 & -1 & -1 \\
\hline$D_{2}$ & 0 & 1 & 0 & -1 & -1 & -1 & -1 \\
\hline$D_{3}$ & 0 & 0 & 0 & 0 & 0 & 0 & 0 \\
\hline$D_{4}$ & 0 & 1 & 1 & 0 & 1 & -1 & -1 \\
\hline
\end{tabular}

From Table 4, we can easily select $D_{3}$ as the transmitter. The vertices of $\mathrm{G}_{L C-I D N C}(\mathcal{V}, \mathcal{E})$ is: $v_{111}^{3}, v_{113}^{3}, v_{215}^{3}, v_{122}^{3}, v_{142}^{3}, v_{143}^{3}, v_{245}^{3}$. And we can generate the edge by the three constrain conditions 1)-3):

The first case of constraint condition 3):

1) $i=i^{\prime}$

2) $l=l^{\prime}$, we can find: $v_{122}^{3}-v_{142}^{3}, v_{113}^{3}-v_{143}^{3}, v_{215}^{3}-v_{245}^{3}$;

3) $p=p^{\prime}$

The second case of constraint condition 3):

1) $i=i^{\prime}$

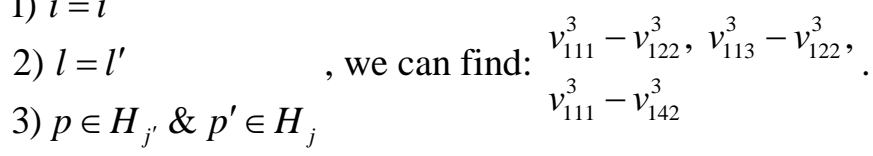

So, the layer cooperation IDNC graph is as Fig. 8:

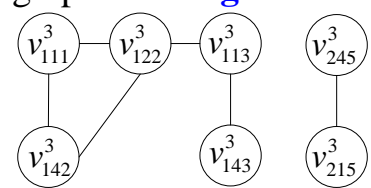

Fig. 8. The layer cooperation IDNC graph

\subsection{Expanded LCID Algorithm by Adding Window}

Since all the devices need the base-layer packets, we constructed window to ensure the transmission priority of $\mathcal{L}_{1}$ packets. We use matrix 'Win-Base' to store the feedback data of receivers about the $\mathcal{L}_{1}$ layer packets. The size of this matrix is the number of receivers times the number of packets of $\mathcal{L}_{1}$. Firstly, the window is initially set to Win-Base. Until the matrix of Win-Base is equal to zero, i.e. all the receivers successfully received the packets of base-layer, the window will expand to the enhancement layers, which is illustrated as Fig. 9. We denote this expanding LCID algorithm as LCIDW.

\begin{tabular}{|c|c|}
\hline $\begin{array}{c}\text { Win- } \\
\text { Base }\end{array}$ & Expanded window \\
\hline $\mathcal{L}_{1}$ & $\mathcal{L}_{2}, \ldots$ \\
\hline
\end{tabular}

Fig. 9. Adding window to layers 


\section{Experimental Results and Analysis}

In this simulation, we utilize the Random Waypoint Mobility model to build the mobile opportunistic device-to-device network, the average speed of devices is set to $8(\mathrm{~m} / \mathrm{s})$, walk time interval is set to [2 4](s), the pause time interval is set to [1 3] (s), and the direction is selected from [-180 180](degree). The packets are divided into three layers. The first layer is the base-layer which are wanted by all the devices, and the other two layers are enhancement layers. Each device requires different layers of packets depending on its computing capability. Since the device-to-device communication is more reliable than the BS broadcast communications [28], the erasure probability $P_{b}$ of BS is set to $2 \times P_{d}$, where $P_{d}$ is the erasure probability of D2D communication [29].

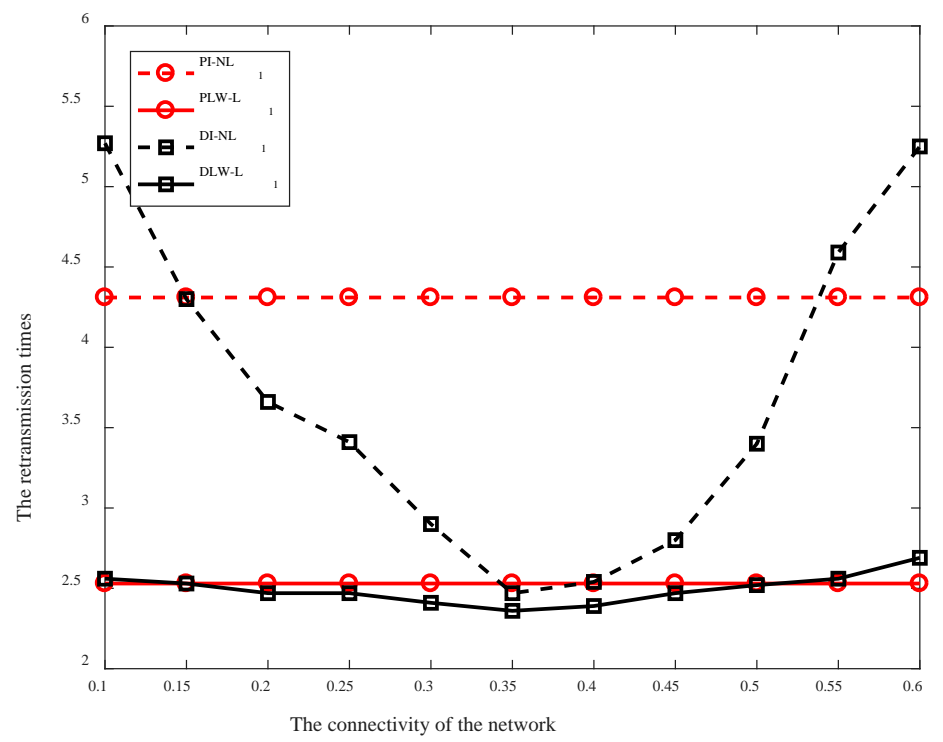

Fig. 10. Retransmission times versus connectivity for a network of 20 packets (in which the base-layer is set to 10 packets), 20 devices, average moving speed: $8 \mathrm{~m} / \mathrm{s}$, BS to device channel erasure probability: 0.15

In section 3, it has illustrated that the DMDR algorithm performs better than the DMWT algorithm to select transmitters. So in the following we use the DMDR algorithm to conduct a further analysis. And then find packets combition for each transmitter by using the IDNC algorithm or our proposed LCIDW algorithm. By combining them with the DMDR algorithm the two algorithms are respectively denoted as DI algorithm and DLW algorithm. In case 1, we consider the packets are not layered. In case 2, the packets are divided into three layers, which consists of a base-layer (i.e. $\mathcal{L}_{1}$ ) and two enhancement layers. For all devices, it is the basic requirement to receive the base-layer packets. When the packets layered, we use the LCIDW algorithm to give the priority to transmit the packets of the base-layer.

In Fig. 10, it illustrates the retransmission times of all the devices successfully receive the base-layer packets under case 1 (Non-layered) and case 2 (layered). When the packets layered, we use our proposed LCIDW algorithm to select packets for each transmitter. We compared the traditional IDNC algorithm (denoted as PI-NL ${ }_{1}, \mathrm{PLW}-\mathrm{L}_{1}$ corresponding to case 1 and case 2 respectively) with our proposed algorithm (denoted as DI-NL ${ }_{1}$, DLW- $\mathrm{L}_{1}$ corresponding to case 1 and case 2 respectively). Here, DLW- $\mathrm{L}_{1}$ represents the retransmission times of the base-layer (i.e. $\mathcal{L}_{1}$ ) packets successfully received by all the receivers by using the DMDR 
transmitters-selecting algorithm combined with the LCIDW packets-selecting algorithm under case 2.

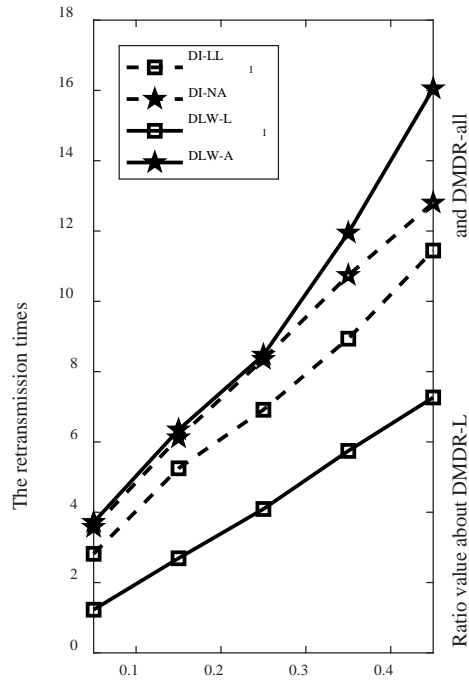

(a) The erasure probability: $\mathrm{p}$

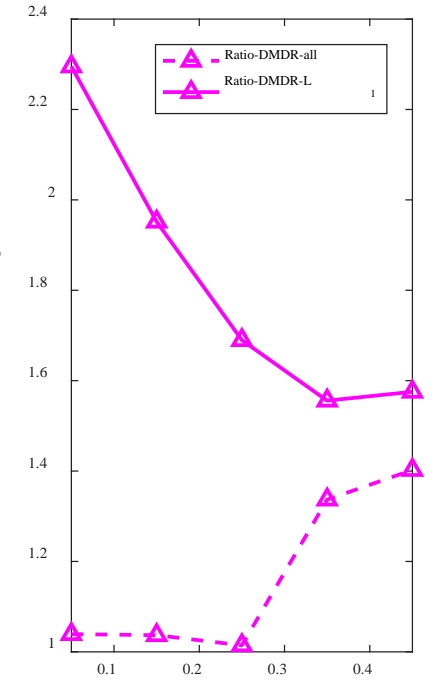

(b) The erasure probability: $\mathrm{p}$

Fig. 11. (a)Retransmission times versus erasure probability of BS broadcast channel for a network of 20 packets (in which the base-layer is set to 10 packets), 20 devices, connectivity: 0.6, average moving speed: $8 \mathrm{~m} / \mathrm{s}$; (b) is the ratio about DMDR-all and DMDR- $\mathrm{L}_{1}$ corresponding to (a)

In case 1 , Fig. 10 depicts the curve of DI-NL ${ }_{1}$ is lower than the PI-NL $\mathrm{N}_{1}$ when the network owns a connectivity in the interval [0.15 0.53] and reach the maximal differentials when the connectivity is 0.35 . However, as Fig. 7 , with the connectivity adding, the curve of DI-NL $\mathrm{N}_{1}$ is going descent firstly and then turns to rise, which is because with the adding of connectivity the packets diffusion capability is enhanced at the beginning. However, when the connectivity is very high, the number of transmitters that can be selected will decrease, which will cause the total retransmission times to increase again.

Besides, from the curves of DI-NL $L_{1}$ and DLW- $\mathrm{L}_{1}$, we see that the DLW algorithm can obtain much less retransmission times than the DI algorithm to ensure the base-layer packets successfully received by all devices regardless of poor or well network connectivity. In addition, DLW performs fewer retransmission times than the PLW algorithm when in case 2 with the connectivity in the interval [0.15 0.5].

Fig. 11. (a) compares the retransmission times of layered and non-layered by using the DMDR algorithm. DLW-NL $L_{1}$ and DLW-A represent the retransmission times that all the devices successfully receive the base-layer packets and the whole layers packets respectively. We can see that the value of DLW- $\mathrm{L}_{1}$ is lower than the other three curves with the increasing of erasure probability.

Fig. 11. (b) can further illustrates the benefits of LCIDW algorithm to select packets combination. The 'Ratio-DMDR-all' is the radio of DLW-A to DI-NA, and the 'Ratio-DMDR-L ' ' is the ratio of DI-NL ${ }_{1}$ to DLW- $\mathrm{L}_{1}$. From Fig. 11. (b) we can conclude that although the DLW algorithm will cause a small scale increase of the total completion decoding times, the times of decoding the base-layer packets by all the devices is reduced drastically, so the advantage outdos the disadvantage a lot. 


\section{Conclusion}

In this paper, we focus on the packets retransmission delay problem of heterogeneous devices in DO-D2D networks. The results of the simulation demonstrate that the proposed DMDR-TS algorithm outperforms the DMWT-TS algorithm in choosing the transmission devices to reduce the retransmission times. Besides, the layer cooperation IDNC algorithm shows it can ensure the base-layer packets successfully received by a higher priority and satisfy the different demands of users with heterogeneous devices. The proposed DMDR-LCIDW algorithm performs better than the traditional PMP-IDNC algorithm for minimizing the packets retransmission times especially in the poor connected and high channel erasure probability network. And how to minimize the retransmission times in the multi-hop relay network also deserves further study.

\section{References}

[1] Forecast C V N I, “Cisco Visual Networking Index: Global Mobile Data Traffic Forecast Update,” 2016-2021 White Paper, Cisco Public Information, March, 2017. Article (CrossRef Link)

[2] M. Deressa Amentie, M. Sheng, J. Song and J. Liu, "Minimum delay guaranteed cooperative device-to-device caching in 5G wireless networks," in Proc. of 2016 8th International Conference on Wireless Communications \& Signal Processing (WCSP), Yangzhou, pp. 1-5, 2016. Article (CrossRef Link)

[3] D. Zhu, C. Xu, J. Qin, Z. Zhou and J. Guan, "Mobility-aware multimedia data transfer using Multipath TCP in Vehicular Network," in Proc. of 2017 13th International Wireless Communications and Mobile Computing Conference (IWCMC), Valencia, pp. 1041-1046, 2017. Article (CrossRef Link)

[4] M. Dong, T. Kimata, K. Sugiura and K. Zettsu, "Quality-of-Experience (QoE) in Emerging Mobile Social Networks," Ieice Transactions on Information \& Systems, vol. 97, no. 10, pp. 2606-2612, 2014. Article (CrossRef Link)

[5] P. Trakas, F. Adelantado, N. Zorba and C. Verikoukis, "A QoE-Aware Joint Resource Allocation and Dynamic Pricing Algorithm for Heterogeneous Networks," in Proc. of GLOBECOM 2017 2017 IEEE Global Communications Conference, Singapore, pp. 1-6, 2017. Article (CrossRef Link)

[6] M. Deressa, M. Sheng, M. Wimmers, J. Liu and M. Mekonnen, "Maximizing Quality of Experience in Device-to-Device Communication Using an Evolutionary Algorithm Based on Users’ Behavior,” IEEE Access, vol. 5, pp. 3878-3888, 2017. Article (CrossRef Link)

[7] D. U. U. Garip, O. Çalık and G. K. Kurt, "Impact of retransmissions on the quality of experience with realistic channel model," in Proc. of 2017 International Symposium on Wireless Communication Systems (ISWCS), Bologna, pp. 299-302, 2017. Article (CrossRef Link)

[8] M. Yu, A. Sprintson, P. Sadeghi, "On minimizing the average packet decoding delay in wireless network coded broadcast," in Proc. of International Symposium on Network Coding (NetCod), Sydney, NSW, pp. 1-5, 2015. Article (CrossRef Link)

[9] Y. Han, H. Wu, Z. Yang and D. Li, "A New Data Transmission Strategy in Mobile D2D Networks-Deterministic, Greedy, or Planned Opportunistic Routing? ," IEEE Transactions on Vehicular Technology, vol. 66, no. 1, pp. 594-609, January, 2017. Article (CrossRef Link)

[10] S. Huang, B. Liang and J. Li, "Distributed Interference and Delay Aware Design for D2D Communication in Large Wireless Networks With Adaptive Interference Estimation,” IEEE Transactions on Wireless Communications, vol. 16, no. 6, pp. 3924-3939, June, 2017. Article (CrossRef Link)

[11] M. Esmaeilzadeh, P. Sadeghi and N. Aboutorab, "Random Linear Network Coding for Wireless Layered Video Broadcast: General Design Methods for Adaptive Feedback-Free Transmission," IEEE Transactions on Communications, vol. 65, no. 2, pp. 790-805, February, 2017. Article (CrossRef Link) 
[12] Y. Keshtkarjahromi, H. Seferoglu, R. Ansari and A. Khokhar, "Content-Aware Network Coding Over Device-to-Device Networks,” IEEE Transactions on Mobile Computing, vol. 16, no. 8, pp. 2147-2158, August, 2017. Article (CrossRef Link)

[13] S. Sorour and S. Valaee, "On Minimizing Broadcast Completion Delay for Instantly Decodable Network Coding," in Proc. of 2010 IEEE International Conference on Communications, Cape Town, pp. 1-5, 2010. Article (CrossRef Link)

[14] M. Jo, T. Maksymyuk, B. Strykhalyuk and C. H. Cho, "Device-to-device-based heterogeneous radio access network architecture for mobile cloud computing," IEEE Wireless Communications, vol. 22, no. 3, pp. 50-58, June, 2015. Article (CrossRef Link)

[15] S. Sorour and S. Valaee, "Completion Delay Minimization for Instantly Decodable Network Codes,” IEEE/ACM Transactions on Networking, vol. 23, no. 5, pp. 1553-1567, October, 2015. Article (CrossRef Link)

[16] A. Douik and S. Sorour, T. Y. Al-Naffouri, H. C. Yang and M. S. Alouini, "Delay reduction in multi-hop device-to-device communication using network coding," 2015 International Symposium on Network Coding (NetCod), Sydney, NSW, pp. 6-10, 2015. Article (CrossRef Link)

[17] M. S. Karim, A. Douik, S. Sorour and P. Sadeghi, "Rate-aware network codes for completion time reduction in device-to-device communications," in Proc. of 2016 IEEE International Conference on Communications (ICC), Kuala Lumpur, pp. 1-7, 2016. Article (CrossRef Link)

[18] A. Orsino et al., "Effects of Heterogeneous Mobility on D2D- and Drone-Assisted Mission-Critical MTC in 5G,” IEEE Communications Magazine, vol. 55, no. 2, pp. 79-87, February, 2017. Article (CrossRef Link)

[19] S. Huang, E. Izquierdo and P. Hao, “Adaptive packet scheduling for scalable video streaming with network coding," in Proc. of 2016 IEEE International Conference on Communications (ICC), Kuala Lumpur, pp. 1-6, 2016. Article (CrossRef Link)

[20] P. Ostovari, J. Wu, A. Khreishah and N. B. Shroff, "Scalable Video Streaming with Helper Nodes Using Random Linear Network Coding,” in IEEE/ACM Transactions on Networking, vol. 24, no. 3, pp. 1574-1587, June, 2016. Article (CrossRef Link)

[21] P. Erdős and L. Lovász, "Problems and results on 3-chromatic Hypergraphs and some related questions,” Infinite and finite sets, pp. 609-627, 1975. Article (CrossRef Link)

[22] Y. Peng, H. Peng, Q. Tang, et al., "An extension of the Motzkin-Straus theorem to non-uniform hypergraphs and its applications,” Discrete Applied Mathematics, pp. 170-175, 2016. Article (CrossRef Link)

[23] D. A. Shabanov, “Around Erdős-Lovász problem on colorings of non-uniform hypergraphs”, Discrete Mathematics, vol. 338, no. 11, pp. 1976-1981, 2015. Article (CrossRef Link)

[24] C. Berge, "Hypergraphs: Combinatorics of Finite Sets,” Handbook of New Religions and Cultural Production, pp. 512-552, 1989. Article (CrossRef Link)

[25] S. Sorour and S. Valaee, "On minimizing broadcast completion delay for instantly decodable network coding,” in Proc. of IEEE ICC, pp. 1-5, May, 2010. Article (CrossRef Link)

[26] Z. Qingfu, S. Jianyong and E. Tsang, “An evolutionary algorithm with guided mutation for the maximum clique problem,” IEEE Transactions on Evolutionary Computation, vol. 9, no. 2, pp. 192-200, April, 2005. Article (CrossRef Link)

[27] M. Brunato and R. Battiti, "R-EVO: A Reactive Evolutionary Algorithm for the Maximum Clique Problem,” IEEE Transactions on Evolutionary Computation, vol. 15, no. 6, pp. 770-782, December, 2011. Article (CrossRef Link)

[28] X. Xu, Y. Zhang, Z. Sun, Y. Hong and X. Tao, “Analytical Modeling of Mode Selection for Moving D2D-Enabled Cellular Networks,” IEEE Communications Letters, vol. 20, no. 6, pp. 1203-1206, June, 2016. Article (CrossRef Link)

[29] N. Aboutorab and P. Sadeghi, "Instantly Decodable Network Coding for Completion Time or Decoding Delay Reduction in Cooperative Data Exchange Systems," IEEE Transactions on Vehicular Technology, vol. 65, no. 3, pp. 1212-1228, March, 2016. Article (CrossRef Link) 

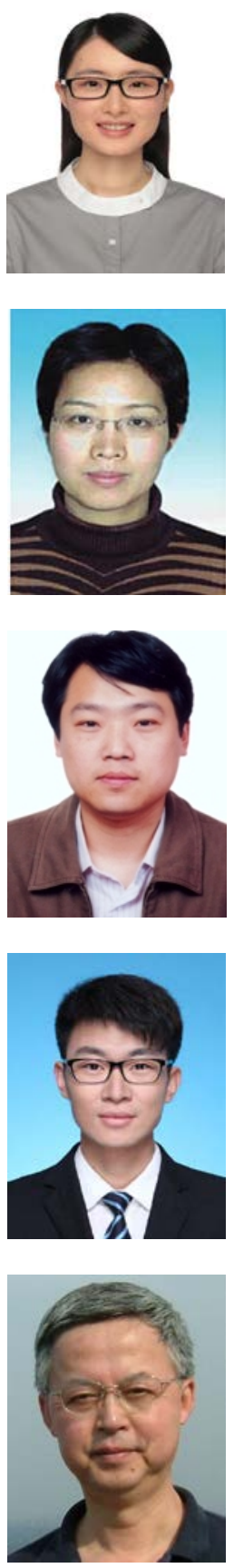

Sixuan Chen received the M.E. degree in Information and Communication Engineering from Chongqing University of Posts and Telecommunications, China, in 2013. She is currently working toward the Ph.D. degree at the Department of Information and Communication Engineering, Beijing University of Posts and Telecommunications. Her research interests include network coding and short-range wireless communications.

Weixia Zou is currently an Associate Professor in Beijing University of Posts and Telecommunications Beijing, China. She received her B.S. degree in electric traction and drive control from Tongji University in 1994, and her M.S. degree in circuits and systems from Shandong University, in 2002, and her Ph.D. degree in communication and information system from Beijing University of Posts and Telecommunications, in 2006. Her current research interests are focused on new technologies of short-range wireless communication and 60GHz.

Xuefeng Liu received the B.S. degree in electrical information engineering from Qufu Normal University in 2002, the M.S. degree in communication and information engineering from Xian University of Science and Technology in 2006. He is now studying at Beijing University of Posts and Telecommunications for a Ph.D. degree in communication and information system. His current research interests are focused on short-range wireless communications, channel modeling, and multi-antenna communication technique.

Yang Zhao received the B.S. degree in communication engineering from Jilin University, China, in 2011. He is currently a master in Beijing University of Posts and Telecommunications. His research interests include network coding and Millimeter wave.

Zheng Zhou received his master's and Ph.D. degrees in electrical engineering from the Beijing University of Posts and Telecommunications (BUPT), China, in 1982 and 1988, respectively. He was the Vice-Dean with the School of Telecommunication Engineering, BUPT, from 1998 to 2003, and was the invited overseas Researcher with the Japan Kyocera DDI Future Communication Research Institute (supported by the Japan Key Technology Center) in 2000. He is currently a Professor with the Beijing University of Posts and Telecommunications, Beijing, China. He was a member of the Technical Subcommittee on Cognitive Networks, the IEEE Communications Society, the International Steering Committee Member of the IEEE International Symposium on Communications and Information Technologies (ISCIT) from 2003 to 2010, and the TPC Co-Chair of the IEEE ISCIT 2005. He was also the General Vice Chair of the IEEE ChinaCom 2006 (the first international conference on communications and networking in China), and the Steering Committee Member of the IEEE ChinaCom 2007. He is a Voting Member and Contributor of the IEEE 802.15 Task Group (TG3a and TG4a), a Senior Member of the China Institution of Communications (CIC), the Radio Application and Management Technical Committee Member of CIC, a Senior Member of the China Computer Federation (CCF), a Sensor Network Technical Committee Member of CCF, an H-Subcommittee Member of the China Radio Interference Standard Technology Committee, the General Secretary of the China UWB Forum, and the General Secretary of the China Bluetooth Forum. 